\title{
Protective Effect of Genipin on Oxidative Stress Injury of Rpe Cells in High Glucose Environment
}

\author{
Shi Yan ${ }^{1 *}$, Chen Qingyou ${ }^{2}$, Xu Wenshuang ${ }^{3}$, Jia Di ${ }^{1}$, Xu Jing ${ }^{1}$ \\ ${ }^{1}$ Department of Biochemistry of Qiqihar Medical College, Qiqihar, Heilongjiang 161000, China \\ ${ }^{2}$ The Third Affiliated Hospital Qiqihar Medical College, Qiqihar, Heilongjiang 161000, China \\ ${ }^{3}$ The Hospital of Qiqihar Otorhinolaryngology, Qiqihar, Heilongjiang 161000, China \\ *Corresponding author
}

Keywords: Genipin, Diabetic retinopathy, Oxidative damage, High glucose

\begin{abstract}
To study the antioxidant effect of Genipin (Gen), an extract from Gardenia jasminoides Ellis, on human Retinal Pigment Epithelium (RPE) cells injured by high glucose.RPE cell injury model was established invitro under high concentration of glucose (50 mmol / L), and the cells were cultured and detected in groups. The activity of superoxide dismutase (SOD) and the content of malondialdehyde (MDA) were measured by WST-1 method and enzyme labeling method, the content of catalase (CAT) was detected by microplate method, and the level of reactive oxygen species (ROS) was detected by fluorescence probe DCF.the content of MDA in the high glucose group (HG) was significantly lower than that in the Genipin plus high glucose group (HG + GEN) $(\mathrm{P}<0.05)$, the activity of antioxidant enzymes SOD, the contents of CAT and GPX were significantly increased $(\mathrm{P}<0.05)$, and the level of ROS detected by DCF fluorescence probe was decreased $(\mathrm{P}<0.05)$. Genipin has protective effect on RPE cells damaged by high concentration glucose.
\end{abstract}

\section{Introduction}

Diabetic retinopathy (DA) is a sign of retinal neovascularization and fibroplasia in hyperglycemia. Retinal pigment epithelium (RPE) cells are one of the cell components in the proliferative membrane of retinopathy. They constitute the external barrier of retina. They are rich in mitochondria and need a lot of glucose for energy supply. They are sensitive to high glucose stimulation. They are vulnerable to oxidative damage of hyperglycemia and affect the function of retinal barrier. Therefore, antioxidant therapy is an effective way to prevent and treat Diabetic retinopathy (DA). Genipin is an active substance from geniposide extracted from Gardenia jasminoides Ellis by $\beta$ - glucosidase hydrolysis. It is easy to enter blood and directly act on target cells. Studies have shown that Genipin has many pharmacological effects, such as antioxidant stress, anti-inflammatory, anti-tumor, reducing blood glucose, improving glucose tolerance and so on ${ }^{1}$. With a good antagonistic effect on the oxidative stress injury of myocardial cells induced by high glucose, Genipin can effectively reduce the oxidative stress injury of liver cells and alleviate the symptoms of spinal cord cerebellar ataxia caused by oxidative stress injury ${ }^{2}$. However, there are few reports about the antioxidant effect of Genipin on RPE cells damaged by high glucose. On the basis of previous studies, we established a model of RPE cell injury induced by high glucose to prove the anti-oxidative effect of Genipin.

\section{Materials and Methods}

\subsection{Materials}

Human RPE cells (ATCC company); Genipin(Cayman); Fetal Bovine Serum (GIBCO company); Glucose (Sigma); 0.25\% pancreatin-0.02\% EDTA (Jinuo Biomedical Technology Co., Ltd.); DMEM medium (Thermo), SOD, MDA, CAT Detection Kit (Shanghai biyuntian Biotechnology 
Co., Ltd.); Reactive oxygen species detection kit (Nanjing Jiancheng biological company). $\mathrm{CO}_{2}$ incubator (Thermo); Fluorescence Microscope (Ningbo Yongxin Optical Co., Ltd.); Enzyme Labeled Instrument (Thermo).

\subsection{Methods}

RPE cell culture: RPE cells were routinely cultured in DMEM medium containing $10 \%$ fetal bovine serum in $37^{\circ} \mathrm{C}$ and $5 \% \mathrm{CO}_{2}$ cell incubator.

Experimental group and pretreatment: cells were divided into four groups. (1) Normal glucose concentration control group (NC group): glucose concentration was $5.56 \mathrm{mmol} / \mathrm{L}$; (2) High concentration glucose injury group (HG group): glucose concentration was $50 \mathrm{mmol} / \mathrm{L}$; (3) Normal glucose concentration glucose + genipin group (NC + Gen group): glucose concentration was 5.56 mmol / L + genipin concentration was $10 \mu \mathrm{mol} / \mathrm{L}$; (4) High concentration glucose + genipin group (HG + Gen group): glucose concentration was $50 \mathrm{mmol} / \mathrm{L}+$ genipin concentration was $10 \mu \mathrm{mol} / \mathrm{L}$.

The contents of MDA, SOD and CAT were detected. RPE cells were digested with $0.25 \%$ pancreatin -0.02\% EDTA, and inoculated in $100 \mathrm{~mm}$ culture plate with $2 \times 10^{5}$ cells / $\mathrm{ml}$. After 24 hours of conventional culture, the operation was carried out according to the instructions of the kit. The optical density (OD) value of each group was measured at the corresponding wavelength, and then converted into the content of MDA, SOD activity and CAT content, and the oxidative stress level of each group was compared.

ROS was detected by DCF. RPE cells were seeded on 96 well plate according to $2 \times 10^{4}$ cells per well. After grouping, the cells were operated according to the instructions of reactive oxygen species assay kit. The optical density was measured at $560 \mathrm{~nm}$.

\subsection{Statistical Processing}

SPSS 22.0 statistical software was used for analysis. The data were expressed by mean \pm SD, and SNK-q test was used to test the pairwise comparison between the mean values of each group. $\mathrm{P}$ $<0.05$ indicated that the difference was statistically significant.

\section{Results}

\subsection{Effects of Genipin on Mda, Sod and Cat Contents in Culture Supernatant of Rpe Cells Injured by High Glucose}

$50 \mathrm{mmol} / \mathrm{L}$ glucose for 72 hours. Compared with the normal glucose control group (NC), the content of MDA was significantly increased in the high glucose injury group (HG), and the contents of SOD and CAT were significantly decreased $(\mathrm{P}<0.05)$. Compared with HG group, $10 \mu \mathrm{mol} / \mathrm{L}$ genipin significantly decreased the content of MDA and increased the content of SOD and CAT (P $<0.05)$. The results are shown in Table 1, which shows that genipin can effectively inhibit the oxidative stress damage of RPE cells induced by high glucose, and has protective effect on cells in high glucose environment. There was no significant difference between NC + Gen group and NC group $(\mathrm{P}>0.05)$, which indicated that genipin had no effect on normal glucose treated cells.

Table 1 the Changes Of the Mda,Sod and Cat Levels (Mean \pm Sd. n=6)

\begin{tabular}{|l|l|l|l|}
\hline Treatment & MDA (nmol/mg prot) & SOD (U/mg prot) & CAT (U/mg protin) \\
\hline NC & $25.64 \pm 2.96$ & $208.15 \pm 11.89$ & $15.34 \pm 0.94$ \\
\hline HG & $33.18 \pm 3.61^{*}$ & $169.85 \pm 19.11^{*}$ & $10.17 \pm 0.58^{*}$ \\
\hline NC+GEN & $27.46 \pm 2.07$ & $201.96 \pm 14.54$ & $14.81 \pm 0.67$ \\
\hline HG+GEN & $26.89 \pm 2.07^{\#}$ & $192.61 \pm 13.15^{\#}$ & $13.72 \pm 0.79^{\#}$ \\
\hline
\end{tabular}

* $\mathrm{P}<0.05$ vs NC group; \# $\mathrm{P}<0.05$ vs HG group.

\subsection{Effects of Genipin on Ros Content in Mitochondria of Rpe Cells Induced by High Glucose}

Compared with normal glucose control group (NC), ROS content in high glucose (HG) group was significantly increased $(\mathrm{P}<0.05)$. Compared with the high glucose $(\mathrm{HG})$ group, the high glucose + genipin (HG + GEN) group significantly reduced the content of ROS induced by high 
glucose $(\mathrm{P}<0.05)$, as shown in Figure 1 , while there was no significant difference between the normal glucose + genipin (NC + GEN) group and the normal glucose control group (NC) $(\mathrm{P}>$ 0.05).

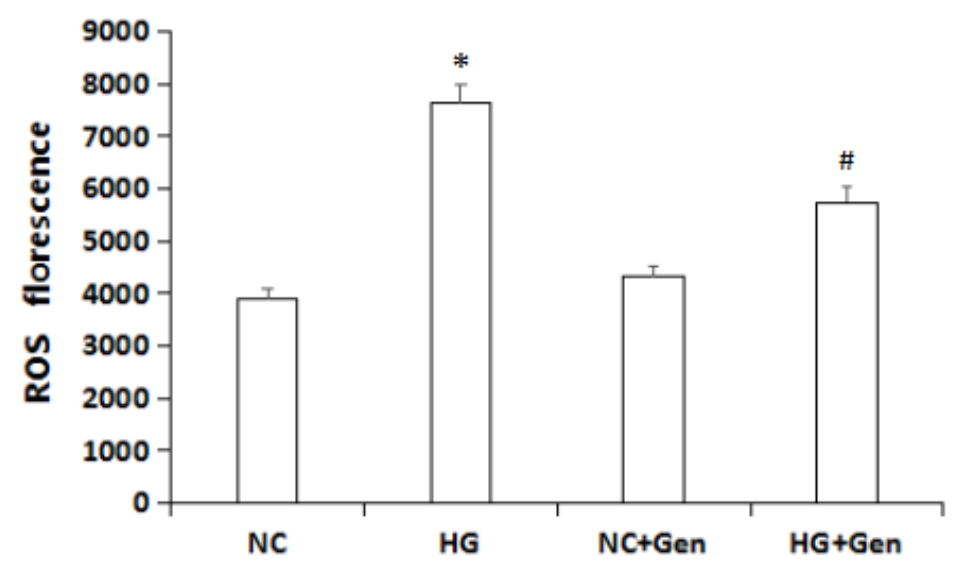

Fig.1 The Effect of Genipin on Ros Production in Rpe Cells Induced by High Glucose. Mean \pm Sd. $\mathrm{n}=3$. * $\mathrm{P}<0.05$ Vs Nc Group; \# $\mathrm{P}<0.05$ Vs Hg Group.

\section{Conclusion}

Diabetic retinopathy (DA) is the most common and serious microvascular complication of diabetes mellitus in the eye. The acute stress response is mainly oxidative stress reaction, changes of signal transduction pathway and apoptosis ${ }^{3}$. Human retinal pigment epithelial cells (RPE) cultured in vitro are the barrier of retina. They simulate the cell-related pathological changes in high glucose environment and the mechanism of genipin. MDA and SOD play an important role in regulating the balance of oxidative stress system and antioxidant kinase. MDA reflects the degree of lipid peroxidation. If the content of MDA is too high, it indicates that the degree of oxidative damage is serious. The activity of SOD can directly reflect the ability of cells to scavenge oxygen free radicals. MDA and SOD mutually verify the oxidative stress state of cells ${ }^{4}$. Antioxidant enzyme CAT further indicated that genipin had protective effect on cells. The results showed that genipin (10 $\mu \mathrm{mol} / \mathrm{L}$ ) could effectively reduce the content of MDA and increase the activity of SOD, which indicated that genipin had obvious protective effect on oxidative stress injury induced by high glucose. Retinal epithelial pigment cells need to consume a lot of oxygen, mainly through the mitochondrial oxidative respiratory chain. High glucose damage can cause a large amount of ROS accumulation, destroy the balance of antioxidant stress system in mitochondria, and lead to mitochondrial oxidative damage. ROS has an important protective effect on the balance of mitochondrial antioxidant stress system, and has become an important indicator to detect the antioxidant enzyme system in mitochondria. In this study, genipin treatment in high glucose environment can significantly reduce ROS production, which further suggests that genipin can reduce the production of intracellular oxidative stress products and maintain the balance of enzymes in mitochondrial antioxidant stress system. In conclusion, genipin has antioxidant effect on retinal pigment epithelial cells damaged by high glucose. Its mechanism of action is to reduce the release of oxidative stress products and balance the antioxidant stress kinase system, which will provide a theoretical basis for the application of new antioxidants in the prevention and treatment of diabetic complications.

\section{References}

[1] Peng Jinjuan, Huang Jiannan, Lu Lina, et al. Research Progress of Remote Screening System for Diabetic Retinopathy. Chinese Journal of Ophthalmology, Vol.52, No.11, pp.868-871, 2016.

[2] Hou Lili, Chen Ting, Liu Liting, et al. Protective Effect of Growth Hormone Releasing Polypeptide on High Glucose Induced Retinal Pigment Epithelial Cells. International Journal of 
Ophthalmology, Vol.18, No.7, pp.1184-1187, 2018.

[3] Zheng Haiyan. Evaluation of Oxidative Stress Injury and Epithelial Mesenchymal Transition of Human Retinal Pigment Epithelial Cells under High Glucose. Journal of Hainan Medical College, Vol.23, No.4, pp.566-569, 2017.

[4] Fan Yan, Lu Hong, Hou Dingshan, et al. Proliferation Promoting and Anti apoptotic Effects of Sphingosine 1-phosphate on Human Retinal Pigment Epithelial Cells under Hypoxia [J]. Chinese Journal of Experimental Ophthalmology, Vol.33, No.1, pp.33-37, 2015. 